\title{
A Lagrangian Dual Approach to the Single-Source Localization Problem
}

\author{
Hou-Duo Qi, Naihua Xiu, and Xiaoming Yuan
}

\begin{abstract}
The single-source localization problem (SSLP), which is nonconvex by its nature, appears in several important multidisciplinary fields such as signal processing and the global positioning system. In this paper, we cast SSLP as a Euclidean distance embedding problem and study a Lagrangian dual approach. It is proved that the Lagrangian dual problem must have an optimal solution under the generalized Slater condition. We provide a sufficient condition for the zero-duality gap and establish the equivalence between the Lagrangian dual approach and the existing Generalized Trust-Region Subproblem (GTRS) approach studied by Beck et al. [3]. We also reveal new implications of the assumptions made by the GTRS approach. Moreover, the Lagrangian dual approach has a straightforward extension to the multiple-source localization problem. Numerical simulations demonstrate that the Lagrangian dual approach can produce localization of similar quality as the GTRS and can significantly outperform the well-known semidefinite programming solver SNLSDP for the multiple source localization problem on the tested cases.
\end{abstract}

\section{Index Terms}

Euclidean distance matrix, Lagrangian duality, orthogonal projection, low-rank approximation.

\section{INTRODUCTION}

The single-source localization problem (SSLP) appears in many important applications including the mobile phones localization [26], [7], [25], localization of the wireless E911 calls [4], and the GPS localization [2] (to name just a few applications). The most often studied criteria for mathematically formulating SSLP are the maximum likelihood (ML) criterion and the least-squares (LS) in the squared domain [4, Sect. 1.2]. Both ML and LS formulations are nonconvex. While the ML criterion has the property of a statistical inference, the LS has the unrivaled property that it can be solved to its global optimality. This is a rare property for nonconvex problems and

Manuscript received October 10, 2012; revised: February 18 and May 15, 2013. This work is supported in part by the National Natural Science Foundation of China (71271021), Engineering and Physical Science Research Council (UK) project EP/K007645/1, and the General Research Grant from Hong Kong Research Grants Council: 203712.

Copyright (c) 2012 IEEE. Personal use of this material is permitted. However, permission to use this material for any other purposes must be obtained from the IEEE by sending a request to pubs-permissions@ieee.org.

H.-D. Qi is with the School of Mathematics, University of Southampton, Highfield, Southampton SO17 1BJ, UK. E-mail: hdqi@soton.ac.uk.

N. Xiu is with the Department of Applied Mathematics, Beijing Jiaotong University, Beijing, China. E-mail: naihua_xiu@126.com.

X. Yuan is with the Department of Mathematics, Hong Kong Baptist University, Kowloon Tong, Hong Kong. E-mail: xmyuan@hkbu.edu.hk. 
was first revealed by Beck et al. [3]. Moreover, the subsequent papers [4], [2] show that this property of the LS formulation plays a key role in designing efficient iterative algorithms for the ML formulation. In this paper, we will focus on the LS formulation.

An important approach that emerged from [3] is to reformulate the LS problem as a Generalized Trust-Region Subproblem (GTRS), which has just one constraint of quadratic equation. It is known that the GTRS possesses a necessary and sufficient condition that characterizes its optimal solutions [21], [11]. Beck et al. [3] studied in detail when the GTRS can be effectively and practically solved to its global optimality.

Another group of important contributions to source localization problems come from the Euclidean distance embedding for sensor network localization, which can be cast as a multiple-source localization problem (see the nice surveys [18], [20] and the references therein). An outstanding feature in this group of papers is that the embedding problem can be related to the celebrated semi-definite programming (SDP), see [1], [5], [28]. The embedding problem can also be relaxed to the nearest Euclidean distance matrix problem [17], [12], [14], [23] and it can also be tackled directly through a Lagrangian dual approach with a majorized penalty technique [24].

However, there is lack of study whether the research from the Euclidean distance embedding has any enhanced/favourable properties for SSLP. This paper tries to initiate such a study by exploring the relationship between the Lagrangian dual approach and the GTRS approach. The major contributions of this paper are summarized as follows.

(i) We initiate the study of the Lagrangian dual approach to the SSLP. We develop a new set of mathematical tools showing that the Lagrangian dual approach is well defined (e.g., the dual problem must admit an optimal solution) under the generalized Slater condition. Those tools also allow us to characterize a sufficient condition for the zero-duality gap to hold. We further establish the equivalence of these two approaches, which are seemingly unrelated while having surprising connections because of our results. Also revealed is a new geometrical interpretation of the assumptions used in the GTRS.

(ii) The Lagrangian dual approach leads to an unconstrained convex optimization, which can be efficiently solved by the well-known HANSO package [22], [19]. Moreover, the Lagrangian dual approach can handle more constraints such as the fixed-distance constraint (45), which would enforce one more quadratic constraint to the GTRS. In contrast, the global theory and the corresponding numerical methods of GTRS remain to be investigated for such cases.

(iii) The application of the Lagrangian dual approach to the multiple-source localization problem is investigated. The advantage of the approach is numerically demonstrated by comparison with the well-known semidefinite programming solver SNLSDP developed by Biswas et al. [5]. On some standard test problems from [5], [29], the Lagrangian dual approach requires only a small fraction of the cpu time of SNLSDP solver and yet provides the localizations of similar quality.

In the following, we describe the basic model of the SSLP. Suppose we have a network of $m$ known sensors (often known as anchors in the literature of sensor network localization), whose coordinates are $\mathbf{a}_{i}=\mathbf{x}_{i} \in \Re^{r}$, $i=1, \ldots, m$. Suppose there is single unknown source, whose coordinate in $\Re^{r}$ is denoted as $\mathbf{x} \in \Re^{r}$. From the 
unknown source, each anchor $\mathbf{a}_{i}$ receives a signal, which can be converted to Euclidean distance $r_{i}$ between $\mathbf{a}_{i}$ and x, possibly with contaminated noise $\epsilon_{i}$ :

$$
r_{i}=\left\|\mathbf{a}_{i}-\mathbf{x}\right\|+\epsilon_{i}, \quad i=1, \ldots, m .
$$

The SSLP is to find $\mathrm{x}$ that solves

$$
\min _{\mathbf{x} \in \Re^{r}} \sum_{i=1}^{m}\left(\left\|\mathbf{x}-\mathbf{a}_{i}\right\|^{2}-r_{i}^{2}\right)^{2} .
$$

We note that problem (1) is the formulation of the squared-range-based least squares considered in [3, Sect. B] and is further called the least squares problem in the squared domain [4, Eq. (1.3)]. We will study a Lagrangian dual approach for (1) and establish its links to the GTRS.

The paper is organized as follows. In the next section, we provide a brief background on Euclidean distance embedding. In Sect. III, we study the Lagrangian dual approach. We first reformulate the SSLP as a Euclidean embedding problem, followed by deriving the Lagrangian dual problem. We then prove that the dual problem has an optimal solution under the generalized Slater condition (Prop. 3.4). A sufficient condition is provided for the zero-duality gap result in Thm. 3.6. In Sect. IV, we establish the equivalence between the Lagrangian dual approach and the GTRS. Numerical results in Sect. V show that the Lagrangian dual approach yields localization of similar quality as that by the GTRS. Moreover, we demonstrate its application to the multiple-source localization problem and show its efficiency by comparison with the popular semidefinite programming solver SNLSDP of [5]. We conclude in Sect. VI.

\section{BACKground on EuclideAn Distance Embedding}

There are three elements that have become basics in the research of Euclidean distance embedding. The first one is the definition of the squared Euclidean distance matrix (EDM). The second are various characterizations of EDMs. And the third one is the Procrustes analysis that produces the actual embedding in a Euclidean space. We briefly describe them one by one. Standard references are [9], [6], [10].

(a) Squared EDM. Let $\mathcal{S}^{n}$ denote the space of $n \times n$ symmetric matrices equipped with the standard inner product $\langle A, B\rangle=\operatorname{trace}(A B)$ for $A, B \in \mathcal{S}^{n}$. Let $\|\cdot\|$ denote the induced Frobenius norm. Let $\mathcal{S}_{+}^{n}$ denote the cone of positive semidefinite matrices in $\mathcal{S}^{n}$ (often abbreviated as $X \succeq 0$ for $X \in \mathcal{S}_{+}^{n}$ ). The so-called hollow subspace $\mathcal{S}_{h}^{n}$ is defined by (“:=” means define)

$$
\mathcal{S}_{h}^{n}:=\left\{A \in \mathcal{S}^{n}: \operatorname{diag}(A)=0\right\},
$$

where $\operatorname{diag}(A)$ is the vector formed by the diagonal elements of $A$. A matrix $D$ is a (squared) EDM if $D \in \mathcal{S}_{h}^{n}$ and there exist points $\mathbf{p}_{1}, \ldots, \mathbf{p}_{n}$ in $\Re^{r}$ such that $D_{i j}=\left\|\mathbf{p}_{i}-\mathbf{p}_{j}\right\|^{2}$ for $i, j=1, \ldots, n$. $\Re^{r}$ is often referred to as the embedding space and $r$ is the embedding dimension when it is the smallest such $r$. All vectors are treated as column vectors. $x^{T}$ means the transpose of the vector $x$, hence it is a row vector.

We note that $D$ must belong to $\mathcal{S}_{h}^{n}$ if it is an EDM. We let $\mathcal{E}^{n}$ be the set of all EDMs in $\mathcal{S}^{n}$, and $\mathcal{E}^{n}(r)$ be the set of all EDMs in $\mathcal{S}^{n}$ with embedding dimensions not greater than $r$. 
(b) Characterizations of EDM. It is well-known that a matrix $D \in \mathcal{S}^{n}$ is an EDM if and only if

$$
D \in \mathcal{S}_{h}^{n} \quad \text { and } \quad J(-D) J \succeq 0 \text {, with } J:=I-e e^{T} / n,
$$

where $I$ (or $I_{n}$ when the indication of dimension is needed) is the identity matrix in $\mathcal{S}^{n}$ and $e$ is the vector of all ones in $\Re^{n}$. The origin of this result can be traced back to Schoenberg [27] and an independent work [30] by Young and Householder. See also Gower [16] for a nice derivation of (2). Moreover, the corresponding embedding dimension is $r=\operatorname{rank}(J D J)$. Consequently, we have

$$
\mathcal{E}^{n}=\left\{A: A \in \mathcal{S}_{h}^{n} \quad \text { and } \quad-J A J \succeq 0\right\}
$$

and

$$
\mathcal{E}^{n}(r)=\mathcal{E}^{n} \cap\left\{A \in \mathcal{S}^{n}: \operatorname{rank}(J A J) \leq r\right\}
$$

We note that $\mathcal{E}^{n}$ is a closed convex cone and $\mathcal{E}^{n}(r)$ is closed, but not convex.

It is noted that the matrix $J$, when treated as an operator, is the orthogonal projection onto the subspace $e^{\perp}:=$ $\left\{x \in \Re^{n}: e^{T} x=0\right\}$. The characterization (2) simply means that $D$ is an EDM if and only if $D \in \mathcal{S}_{h}^{n}$ and $D$ is negative semidefinite on the subspace $e^{\perp}$ :

$$
-D \in \mathcal{K}_{+}^{n}:=\left\{A \in \mathcal{S}^{n}: x^{T} A x \geq 0, \quad x \in e^{\perp}\right\} .
$$

It follows that $\mathcal{K}_{+}^{n}$ is a closed convex cone.

We therefore have another characterization of $\mathcal{E}^{n}$ :

$$
\mathcal{E}^{n}=\mathcal{S}_{h}^{n} \cap\left(-\mathcal{K}_{+}^{n}\right) .
$$

For $\mathcal{E}^{n}(r)$, we have

$$
\mathcal{E}^{n}(r)=\mathcal{S}_{h}^{n} \cap\left(-\mathcal{K}_{+}^{n}(r)\right)
$$

where

$$
\mathcal{K}_{+}^{n}(r):=\mathcal{K}_{+}^{n} \cap\left\{A \in \mathcal{S}^{n}: \operatorname{rank}(J A J) \leq r\right\} .
$$

(c) Euclidean Embedding. If $D$ is an EDM with embedding dimension $r$, then $-J D J \succeq 0$. Let

$$
-J D J / 2=P P^{T}
$$

where $P \in \Re \Re^{n \times r}$. Let $\mathbf{p}_{i}$ denote the $i$ th column of $P^{T}$. It is known [9] that $\left\{\mathbf{p}_{1}, \ldots, \mathbf{p}_{n}\right\}$ are the embedding points of $D$ in $\Re^{r}$, i.e., $D_{i j}=\left\|\mathbf{p}_{i}-\mathbf{p}_{j}\right\|^{2}$. We also note that any rotation and shifting of $\left\{\mathbf{p}_{1}, \ldots, \mathbf{p}_{n}\right\}$ would give the same $D$. In other words, there are infinitely many sets of embedding points. To find a desired set of embedding points that match positions of the existing anchors, one needs to conduct the Procrustes analysis, which is a simple computational scheme, see [9, Chp. 5]. We omit the details. 


\section{LAGRANGIAN DUAL APPROACH}

In this section, we first cast problem (1) as a Euclidean distance embedding problem. We then derive its Lagrangian dual problem. Finally, we study when the dual problem admits an optimal solution and provides a sufficient condition for the zero-duality gap.

\section{A. SSLP as Euclidean Distance Embedding}

For SSLP (1), we have $m$ known anchors $\left\{\mathbf{a}_{1}, \ldots, \mathbf{a}_{m}\right\}$ and an unknown source $\mathbf{x} \in \Re^{r}$. Define the EDM $Y^{*} \in \mathcal{S}^{n}$ (with $n:=m+1$ ) by (only define the upper triangular part of $Y^{*}$ )

$$
Y_{i j}^{*}:= \begin{cases}\left\|\mathbf{a}_{i}-\mathbf{a}_{j}\right\|^{2} & \text { if } i \leq j=2, \ldots, m \\ \left\|\mathbf{a}_{i}-\mathbf{x}\right\|^{2} & \text { if } j=m+1 .\end{cases}
$$

It follows from (2) that $-J Y^{*} J \succeq 0$ and one set of embedding points $\left\{\mathbf{p}_{1}, \ldots, \mathbf{p}_{n}\right\}$ in $\Re^{r}$ would be the columns of $P^{T}$, where $P \in \Re^{n \times r}$ satisfies

$$
-J Y^{*} J / 2=P P^{T}
$$

Moreover, $\left\{\mathbf{a}_{1}, \ldots, \mathbf{a}_{m}, \mathbf{x}\right\}$ would be obtained from $\left\{\mathbf{p}_{1}, \ldots, \mathbf{p}_{n}\right\}$ through the Procrustes analysis.

However, the only issue with the above procedure is that the distances in the last columns of $Y^{*}$ (the distances between $\mathbf{a}_{i}$ and $\mathbf{x}$ ) are not completely known so that the decomposition of (6) is not possible. We only know the contaminated distance measurement $r_{i}$ between $\mathbf{a}_{i}$ and $\mathbf{x}$. We need to recover the true distances from those measured distances $\left\{r_{i}\right\}$.

Define $D \in \mathcal{S}^{n}$ by

$$
D_{i j}:= \begin{cases}\left\|\mathbf{a}_{i}-\mathbf{a}_{j}\right\|^{2} & \text { if } i \leq j=2, \ldots, m \\ r_{i}^{2} & \text { if } j=m+1 .\end{cases}
$$

Our purpose is to recover $Y^{*}$ from $D$. We know that $Y^{*}$ has to satisfy the following conditions:

$$
Y_{i j}^{*}=D_{i j} \quad \text { for } i<j=2, \ldots, m, \quad Y^{*} \in \mathcal{E}^{n},
$$

and $Y^{*}$ has to have the embedding dimension $r$. We are therefore to recover $Y^{*}$ through the following optimization problem:

$$
\begin{aligned}
Y^{*} & =\arg \min \|Y-D\|^{2} / 2 \\
& \text { s.t. } Y_{i j}=D_{i j}, \quad i<j=2, \ldots, m, Y \in \mathcal{E}^{n}(r) .
\end{aligned}
$$

If $r_{i}=\left\|\mathbf{a}_{i}-\mathbf{x}\right\|$ (true distances), then $Y^{*}=D$ is the original EDM. Otherwise, this problem is to calculate the nearest EDM from $D$ subject to the requirements that the solution must obey the pairwise distances among the known anchors $\left\{\mathbf{a}_{i}\right\}$ and that it has to have an embedding dimension not greater than $r$.

Once the optimal solution $Y^{*}$ of (7) is obtained, we can use (6) and the Procrustes analysis to obtain the actual embedding $\left\{\mathbf{a}_{1}, \ldots, \mathbf{a}_{m}, \mathbf{x}\right\}$. The solution method that we propose in this paper for (7) is the Lagrange dual approach. We will show that this approach has some nice and important properties as mentioned in Introduction. In particular, it has a close relationship with the GTRS. We describe this approach below. 


\section{B. The Lagrangian Dual Problem}

The purpose of this part is to solve (7) through its Lagrangian dual problem. Let us first define a few more notations. Define two linear mappings $\mathcal{A}_{1}: \mathcal{S}^{n} \mapsto \Re^{n}$ and $\mathcal{A}_{2}: \mathcal{S}^{n} \mapsto \Re^{m(m-1) / 2}$ by (we identify a vector $v \in \Re^{m(m-1) / 2}$ by ordering its components as $v_{i j}, i<j=2, \ldots, m$ )

$$
\mathcal{A}_{1}(Y):=\operatorname{diag}(Y) \quad \text { and } \quad\left(\mathcal{A}_{2}(Y)\right)_{i j}:=Y_{i j} .
$$

Correspondingly, define $b^{d} \in \Re^{n}$ and $b^{o} \in \Re^{m(m-1) / 2}$ by

$$
b^{d}:=0 \quad \text { and } \quad b_{i j}^{o}:=D_{i j} \quad \forall i<j=2, \ldots, m .
$$

Let

$$
\mathcal{A}:=\left(\begin{array}{c}
\mathcal{A}_{1} \\
\mathcal{A}_{2}
\end{array}\right) \quad \text { and } \quad b:=-\left(\begin{array}{c}
b^{d} \\
b^{o}
\end{array}\right) .
$$

We let $\mathcal{A}^{*}: \Re^{n} \times \Re^{m \times(m-1) / 2} \mapsto \mathcal{S}^{n}$ be the adjoint of $\mathcal{A}$.

Because of (5), problem (7) is equivalent to

$$
\min \frac{1}{2}\|Y-D\|^{2} \quad \text { s.t. } \quad \mathcal{A}(Y)=-b \text { and }-Y \in \mathcal{K}_{+}^{n}(r),
$$

or equivalently by replacing $Y$ with $-Y$

$$
v_{p}:=\min \frac{1}{2}\|Y+D\|^{2} \text { s.t. } \mathcal{A}(Y)=b \text { and } Y \in \mathcal{K}_{+}^{n}(r) .
$$

The Lagrangian dual function for (9) is

$$
L(Y, y):=\frac{1}{2}\|Y+D\|^{2}+\langle b-\mathcal{A}(Y), y\rangle,
$$

where $Y \in \mathcal{S}^{n}$ and $y \in \Re^{n} \times \Re^{m \times(m-1) / 2}$. The Lagrangian dual problem is then given by

$$
v_{d}:=\max _{y \in \Re^{n} \times \Re^{m \times(m-1) / 2}} \theta(y),
$$

where

$$
\theta(y):=\min _{Y \in \mathcal{K}_{+}^{n}(r)} L(Y, y) .
$$

It always holds that $v_{p} \geq v_{d}$. The quantity $v_{p}-v_{d}$ is called the duality gap. When this quantity vanishes, we say there is no duality gap (i.e., zero-duality gap). We now derive an explicit expression for $\theta(y)$.

Given a closed set $\mathcal{C} \subset \mathcal{S}^{n}$ and $X \in \mathcal{S}^{n}$, let $\Pi_{\mathcal{C}}(X)$ denote an optimal solution of the following metric projection problem:

$$
\min \|Z-X\|^{2} \quad \text { s.t. } Z \in \mathcal{C} .
$$

When $\mathcal{C}$ is convex, $\Pi_{\mathcal{C}}(X)$ is unique. When $\mathcal{C}$ is not convex, there may be multiple solutions and we let $\Pi_{\mathcal{C}}^{B}(X)$ denote the set of all projections of $X$ onto $\mathcal{C}$. Let $\mathcal{S}_{+}^{n}(r)$ be the set of all positive semidefinite matrices whose ranks are not greater than $r$. Both $\mathcal{S}_{+}^{n}(r)$ and $\mathcal{K}_{+}^{n}(r)$ are not convex.

Let $X$ have the spectral decomposition

$$
X=P \Lambda(X) P^{T},
$$


where $\Lambda(X):=\operatorname{Diag}(\lambda(X)), \lambda_{1}(X) \geq \ldots \geq \lambda_{n}(X)$ are the eigenvalues of $X$ being arranged in the non-increasing order and $P \in \mathcal{O}^{n}$ with $\mathcal{O}^{n}$ being the set of all $n \times n$ orthogonal real matrices. Define

$$
\alpha:=\left\{i \mid \lambda_{i}(X)>\lambda_{r}(X)\right\} \text { and } \beta:=\left\{i \mid \lambda_{i}(X)=\lambda_{r}(X)\right\} .
$$

For an index set $\mathcal{I} \subseteq\{1, \ldots, n\}, X_{\mathcal{I}}$ denotes the submatrix consisting of the columns in $X$ indexed by $\mathcal{I}$ and $|\mathcal{I}|$ denotes the cardinality of $\mathcal{I}$.

Lemma 3.1: [13, Lemma 2.4] The set $\Pi_{\mathcal{S}_{+}^{n}(r)}^{B}(X)$ can be characterized as follows.

$$
\Pi_{\mathcal{S}_{+}^{n}(r)}^{B}(X)=\left\{\left[P_{\alpha}, P_{\beta} V_{\beta}\right] \operatorname{Diag}(v)\left[P_{\alpha}, P_{\beta} V_{\beta}\right]^{T} \mid V_{\beta} \in \mathcal{O}^{|\beta|}\right\}
$$

where $v:=\left(\left(\lambda_{1}(X)\right)_{+}, \ldots,\left(\lambda_{r}(X)\right)_{+}, 0, \ldots, 0\right)^{T} \in \Re^{|\alpha|+|\beta|}$.

Lemma 3.2: [24, Prop. 3.3] The set $\Pi_{\mathcal{K}_{+}^{n}(r)}^{B}(X)$ can be characterized as follows.

$$
\Pi_{\mathcal{K}_{+}^{n}(r)}^{B}(X)=\Pi_{\mathcal{S}_{+}^{n}(r)}^{B}(J X J)+(X-J X J) .
$$

Moreover, for any pair $\left(\Pi_{\mathcal{K}_{+}^{n}(r)}(X), \Pi_{\mathcal{S}_{+}^{n}(r)}(J X J)\right)$ satisfying

$$
\Pi_{\mathcal{K}_{+}^{n}(r)}(X)=\Pi_{\mathcal{S}_{+}^{n}(r)}(J X J)+(X-J X J),
$$

we have

$$
\begin{gathered}
\left\|\Pi_{\mathcal{K}_{+}^{n}(r)}(X)\right\|^{2}=\left\|\Pi_{\mathcal{S}_{+}^{n}(r)}(J X J)\right\|^{2}+\|X-J X J\|^{2}, \\
J \Pi_{\mathcal{K}_{+}^{n}(r)}(X) J=\Pi_{\mathcal{S}_{+}^{n}(r)}(J X J),
\end{gathered}
$$

and

$$
\left\langle\Pi_{\mathcal{K}_{+}^{n}(r)}(X), X-\Pi_{\mathcal{K}_{+}^{n}(r)}(X)\right\rangle=0 .
$$

We now derive an explicit formula for $\theta(y)$.

$$
\begin{aligned}
\theta(y)= & \min _{Y \in \mathcal{K}_{+}^{n}(r)} L(Y, y) \\
= & \min _{Y \in \mathcal{K}_{+}^{n}(r)} \frac{1}{2}\|Y+D\|^{2}-\left\langle Y, \mathcal{A}^{*}(y)\right\rangle+\langle b, y\rangle \\
= & \min _{Y \in \mathcal{K}_{+}^{n}(r)} \frac{1}{2}\left\|Y-\left(-D+\mathcal{A}^{*}(y)\right)\right\|^{2} \\
& -\frac{1}{2}\left\|-D+\mathcal{A}^{*}(y)\right\|^{2}+\frac{1}{2}\|D\|^{2}+\langle b, y\rangle \\
= & \frac{1}{2}\left\|\Pi_{\mathcal{K}_{+}^{n}(r)}\left(-D+\mathcal{A}^{*}(y)\right)-\left(-D+\mathcal{A}^{*}(y)\right)\right\|^{2} \\
& -\frac{1}{2}\left\|-D+\mathcal{A}^{*}(y)\right\|^{2}+\frac{1}{2}\|D\|^{2}+\langle b, y\rangle \\
= & -\frac{1}{2}\left\|\Pi_{\mathcal{K}_{+}^{n}(r)}\left(-D+\mathcal{A}^{*}(y)\right)\right\|^{2}+\frac{1}{2}\|D\|^{2}+\langle b, y\rangle .
\end{aligned}
$$

The last equality used (14). Hence, the dual problem (10) becomes (in the form of minimization)

$$
-v_{d}=\min \Theta(y),
$$


where

$$
\Theta(y)=-\theta(y)=\frac{1}{2}\left\|\Pi_{\mathcal{K}_{+}^{n}(r)}\left(-D+\mathcal{A}^{*}(y)\right)\right\|^{2}-\langle b, y\rangle-\frac{1}{2}\|D\|^{2} .
$$

We note that $\Theta(\cdot)$ is convex. In the next section, we study when the problem (15) has an optimal solution and when the duality gap vanishes (i.e., $v_{p}=v_{d}$ ).

\section{Existence of Optimal Dual Solutions and Zero Duality Gap}

Let us consider the linear system

$$
\mathcal{A}(Y)=b \quad \text { and } \quad Y \in \mathcal{K}_{+}^{n},
$$

with $\mathcal{A}$ and $b$ defined in (8). It is obvious that the linear mappings $\mathcal{A}_{1}$ and $\mathcal{A}_{2}$ are linearly independent. We say that the (generalized) Slater condition for (16) holds if there exists $Y^{0} \in \mathcal{K}_{+}^{n}$ such that

$$
Y^{0} \in \operatorname{int}\left(\mathcal{K}_{+}^{n}\right) \quad \text { and } \quad \mathcal{A}\left(Y^{0}\right)=b,
$$

where $\operatorname{int}\left(\mathcal{K}_{+}^{n}\right)$ is the interior of $\mathcal{K}_{+}^{n}$.

The Slater condition is closely related to the polar cone $\left(\mathcal{K}_{+}^{n}\right)^{*}$ of $\mathcal{K}_{+}^{n}$ :

$$
\begin{aligned}
\left(\mathcal{K}_{+}^{n}\right)^{*} & :=\left\{X \in \mathcal{S}^{n}:\langle X, A\rangle \leq 0 \quad \forall A \in \mathcal{K}_{+}^{n}\right\} \\
& =\left\{Q\left[\begin{array}{ll}
Z & 0 \\
0 & 0
\end{array}\right] Q: Z \in-\mathcal{S}_{+}^{n-1}\right\},
\end{aligned}
$$

where the last equality used [23, Eq. (16)] and $Q$ is the Householder matrix defined by

$$
Q:=I-\frac{2}{v^{T} v} v v^{T} \quad \text { with } \quad v:=[1, \ldots, 1, \sqrt{n}+1]^{T} \in \Re^{n} .
$$

The following result is a direct consequence of a result of Gao [13] when applied to (16).

Proposition 3.3: [13, Prop. 2.20] If the Slater condition holds for (16), then $\langle b, y\rangle<0$ for any $0 \neq y \in$ $\Re^{n} \times \Re^{m \times(m-1) / 2}$ satisfying $\mathcal{A}^{*}(y) \in\left(\mathcal{K}_{+}^{n}\right)^{*}$.

The following result shows that the Slater condition guarantees the existence of an optimal solution for the dual problem (15). The proof of the following result is motivated by [13, Prop. 4.16].

Proposition 3.4: Assume that the Slater condition holds for (16). Then the level set $L_{c}:=\left\{y \in \Re^{n} \times \Re^{m(m-1) / 2}\right.$ : $\Theta(y) \leq c\}$ is bounded for any constant $c \in \Re$. Consequently, the Lagrangian dual problem (15) must have an optimal solution.

Proof: We prove the conclusion of this result by contradiction. Suppose that there exists a constant $c$ such that $L_{c}$ is unbounded. There must be a sequence $\left\{y^{k}\right\}$ satisfying $\left\|y^{k}\right\| \rightarrow \infty$ and $\Theta\left(y^{k}\right) \leq c$ for all $y^{k}$. Without loss of generality, we assume that $y^{k} /\left\|y^{k}\right\| \rightarrow \bar{y}$. Denote $B^{k}:=\left(-D+\mathcal{A}^{*}\left(y^{k}\right)\right) /\left\|y^{k}\right\|$. We have $\lim _{k \rightarrow \infty} B^{k}=\mathcal{A}^{*}(\bar{y})$. We consider two separate cases. Case 1: $\mathcal{A}^{*}(\bar{y}) \notin\left(\mathcal{K}_{+}^{n}\right)^{*}$ and Case 2: $\mathcal{A}^{*}(\bar{y}) \in\left(\mathcal{K}_{+}^{n}\right)^{*}$.

Case 1. Denote

$$
Q\left(\mathcal{A}^{*}(\bar{y})\right) Q=:\left[\begin{array}{cc}
\widehat{B}_{1} & \hat{b} \\
\hat{b}^{T} & \hat{b}_{0}
\end{array}\right] \text { with } \widehat{B}_{1} \in \mathcal{S}^{n-1} \text {. }
$$


The Householder matrix $Q$ and the projection matrix $J$ have the nice relationship.

$$
J=Q\left[\begin{array}{cc}
I_{n-1} & 0 \\
0 & 0
\end{array}\right] Q .
$$

It follows that

$$
J\left(\mathcal{A}^{*}(\bar{y})\right) J=Q\left[\begin{array}{cc}
\widehat{B}_{1} & 0 \\
0 & 0
\end{array}\right] Q .
$$

Because $Q^{2}=I$, we have from (21) that

$$
\left\|\Pi_{\mathcal{S}_{+}^{n}(r)}\left(J\left(\mathcal{A}^{*}(\bar{y})\right) J\right)\right\|^{2}=\left\|\Pi_{\mathcal{S}_{+}^{n-1}(r)}\left(\widehat{B}_{1}\right)\right\|^{2}
$$

and

$$
\left\|\mathcal{A}^{*}(\bar{y})-J\left(\mathcal{A}^{*}(\bar{y})\right) J\right\|^{2}=2\|\hat{b}\|^{2}+\hat{b}_{0}^{2}
$$

In this case, $\mathcal{A}^{*}(\bar{y}) \notin\left(\mathcal{K}_{+}^{n}\right)^{*}$. That is (because of (18)),

$$
Q\left(\mathcal{A}^{*}(\bar{y})\right) Q \notin\left\{\left[\begin{array}{ll}
Z & 0 \\
0 & 0
\end{array}\right]: Z \in-\mathcal{S}_{+}^{n-1}\right\} .
$$

It follows from (19) that there are two possibilities. Subcase 1.1: $\widehat{B}_{1} \notin-\mathcal{S}_{+}^{n-1}$ and Subcase 1.2: $\left[\hat{b}^{T}, \hat{b}_{0}\right] \neq[0,0]$. For Subcase 1.1, $\widehat{B}_{1}$ must have at least one positive eigenvalue. Then it follows from (22) that there exists $\delta_{1}>0$ such that

$$
\left\|\Pi_{\mathcal{S}_{+}^{n}(r)}\left(J \mathcal{A}^{*}(\bar{y}) J\right)\right\|^{2}=\left\|\Pi_{\mathcal{S}_{+}^{n-1}(r)}\left(\widehat{B}_{1}\right)\right\|^{2} \geq \delta_{1} .
$$

For Subcase 1.2, there exists $\delta_{2}>0$ such that

$$
\left\|\mathcal{A}^{*}(\bar{y})-J \mathcal{A}^{*}(\bar{y}) J\right\|^{2}=2\|\hat{b}\|^{2}+\hat{b}_{0}^{2} \geq \delta_{2} .
$$

By the continuity of $\left\|\Pi_{\mathcal{K}_{+}^{n}(r)}(\cdot)\right\|^{2}$ (due to it being convex, see Lemma 3.5 below), we have

$$
\begin{aligned}
& \lim _{k \rightarrow \infty}\left\|\Pi_{\mathcal{K}_{+}^{n}(r)}\left(B^{k}\right)\right\|^{2} \\
= & \lim _{k \rightarrow \infty}\left(\left\|\Pi_{\mathcal{S}_{+}^{n-1}(r)}\left(J B^{k} J\right)\right\|^{2}+\left\|B^{k}-J B^{k} J\right\|^{2}\right) \\
= & \left\|\Pi_{\mathcal{S}_{+}^{n}(r)}\left(J \mathcal{A}^{*}(\bar{y}) J\right)\right\|^{2}+\left\|\mathcal{A}^{*}(\bar{y})-J \mathcal{A}^{*}(\bar{y}) J\right\|^{2} \\
\geq & \min \left\{\delta_{1}, \delta_{2}\right\}>0,
\end{aligned}
$$

where the first equation used (12). Hence, we have

$$
\begin{aligned}
& \lim _{k \rightarrow \infty} \Theta\left(y^{k}\right) \\
= & \lim _{k \rightarrow \infty}\left(\frac{1}{2}\left\|\Pi_{\mathcal{K}_{+}^{n}(r)}\left(-D+\mathcal{A}^{*}\left(y^{k}\right)\right)\right\|^{2}-\left\langle b, y^{k}\right\rangle\right) \\
\geq & \left.\lim _{k \rightarrow \infty}\left\|y^{k}\right\|\left(\frac{1}{2}\left\|y^{k}\right\|\left\|\Pi_{\mathcal{K}_{+}^{n}(r)}\left(B^{k}\right)\right\|^{2}-\|b\|\right)\right)=+\infty .
\end{aligned}
$$


Case 2. We note that $\bar{y} \neq 0$. It follows from Prop. 3.3 that $\langle b, \bar{y}\rangle<0$. Therefore,

$$
\begin{aligned}
\lim _{k \rightarrow \infty} \Theta\left(y^{k}\right) & \geq \lim _{k \rightarrow \infty}\left\|y^{k}\right\|\left(-\left\langle b, y^{k} /\left\|y^{k}\right\|\right\rangle\right) \\
& \geq-\frac{\langle b, \bar{y}\rangle}{2} \lim _{k \rightarrow \infty}\left\|y^{k}\right\|=+\infty
\end{aligned}
$$

Either of the cases contradicts our assumption that $\left\{\Theta\left(y^{k}\right)\right\}$ is bounded. This contradiction establishes the boundedness of the level set.

The generalized Slater condition is a standard assumption in optimization. It is worth noting that the condition (17) is equivalent to the following Euclidean distance matrix completion problem: There exists Euclidean distance matrix $Y$ such that $Y_{i j}=D_{i j}$ for $i<j=2, \ldots, m$ and the embedding dimension of $Y$ is $(n-1)$. We omit its proof. However, it is also worth mentioning that there may exist other EDM $\bar{Y}$ such that $\bar{Y}_{i j}=D_{i j}$ for $i<j=2, \ldots, m$ with its embedding dimension strictly less than $(n-1)$. We do not know whether the generalized Slater condition is also necessary for Prop. 3.4. Next we present our zero duality gap result, which needs the following result.

Lemma 3.5: [24, Prop. 3.4] Let

$$
\Xi_{r}^{e}(Z):=\frac{1}{2}\left\|\Pi_{\mathcal{K}_{+}^{n}(r)}(Z)\right\|^{2}, \quad \forall Z \in \mathcal{S}^{n} .
$$

Then $\Xi_{r}^{e}(\cdot)$ is convex and its subdifferential is the convex hull of $\Pi_{\mathcal{K}_{+}^{n}(r)}^{B}(Z)$ :

$$
\partial \Xi_{r}^{e}(Z)=\operatorname{conv}\left\{\Pi_{\mathcal{K}_{+}^{n}(r)}^{B}(Z)\right\}
$$

Theorem 3.6: Suppose that $\bar{y}$ is an optimal solution of the dual problem (15). Let $\lambda_{1} \geq \lambda_{2} \geq \ldots \geq \lambda_{n}$ be the eigenvalues of $J\left(-D+\mathcal{A}^{*}(\bar{y})\right) J$. If $\lambda_{r+1} \leq 0$ or in the case of $\lambda_{r+1}>0, \lambda_{r}>\lambda_{r+1}$, then $\Pi_{\mathcal{K}_{+}^{n}(r)}^{B}\left(-D+\mathcal{A}^{*}(\bar{y})\right)$ contains just one element, denoted by $\bar{Y}:=\Pi_{\mathcal{K}_{+}^{n}(r)}\left(-D+\mathcal{A}^{*}(\bar{y})\right)$. Moreover, $\bar{Y}$ globally solves the problem (9) and the zero duality holds.

Proof: Since $\Theta(\cdot)$ is convex, $\bar{y}$ is an optimal solution of (15) if and only if $0 \in \partial \Theta(\bar{y})$. We note that $\Theta(\cdot)$ is composition of $\Xi_{r}^{e}(\cdot)$ and $\mathcal{A}^{*}$, i.e.,

$$
\Theta(y)=\Xi_{r}^{e}\left(-D+\mathcal{A}^{*}(y)\right)-\langle b, y\rangle-\frac{1}{2}\|D\|^{2} .
$$

By the chain rule of the subdifferential, we have

$$
\begin{aligned}
\partial \Theta(y)= & \mathcal{A}\left(\partial \Xi_{r}^{e}\left(-D+\mathcal{A}^{*}(y)\right)\right)-b \\
= & \mathcal{A}\left(\operatorname{conv}\left\{\Pi_{\mathcal{K}_{+}^{n}(r)}^{B}\left(-D+\mathcal{A}^{*}(y)\right)\right\}\right)-b \\
= & \mathcal{A}\left(\operatorname{conv}\left\{\Pi_{\mathcal{S}_{+}^{n}(r)}^{B}\left(J\left(-D+\mathcal{A}^{*}(y)\right) J\right)\right\}\right. \\
& \left.-\left(-D+\mathcal{A}^{*}(y)-J\left(-D+\mathcal{A}^{*}(y)\right) J\right)\right)-b
\end{aligned}
$$

The second equality used Lemma 3.5 and the last equality used Lemma 3.2.

Under the condition in the theorem, it is easy to verify from Lemma 3.1 that $\Pi_{\mathcal{S}_{+}^{n}(r)}^{B}\left(J\left(-D+\mathcal{A}^{*}(\bar{y})\right) J\right)$ is singleton. This implies that $\partial \Theta(\bar{y})$ is singleton. The optimality condition $0 \in \partial \Theta(\bar{y})$ means

$$
b=\mathcal{A}(\bar{Y})
$$


and $\bar{Y}=\Pi_{\mathcal{K}_{+}^{n}(r)}\left(-D+\mathcal{A}^{*}(y)\right) \in \mathcal{K}_{+}^{n}(r)$. Therefore, $\bar{Y}$ is a feasible point of (9). Moreover, we have

$$
\begin{aligned}
v_{d} & \geq \Theta(\bar{y})=L(\bar{Y}, \bar{y})=\frac{1}{2}\|\bar{Y}+D\|^{2}+\langle b-\mathcal{A}(\bar{Y}), \bar{y}\rangle \\
& =\frac{1}{2}\|\bar{Y}+D\|^{2} \geq v_{p} .
\end{aligned}
$$

This proves $v_{d}=v_{p}$ because we always have $v_{p} \geq v_{d}$. In other words, $\bar{Y}$ globally solves the problem (9) and the zero duality gap holds.

The dual problem is convex and the subdifferential of $\Theta(\cdot)$ can be calculated through (23) and the characterization of $\Pi_{\mathcal{S}_{+}^{n}(r)}^{B}(X)$ in Lemma 3.1. The famous HANso package [22], [19] can be used to solve (15). As mentioned in the introduction, another global method is the GTRS contributed by Beck et al. [3]. It is surprising that these two approaches are actually equivalent under reasonable conditions. It is proved in the next section.

\section{Equivalence to GTRS}

In this section, we first state the GTRS reformulation by Beck et al. [3], its global optimality condition, and the assumptions that ensure an efficient solution method of the GTRS. We also study some implications of those assumptions. We then establish the equivalence between these two approaches.

\section{A. GTRS and Its Global Optimality Condition}

Beck et al. [3, Sect. B] reformulated this problem as the Generalized Trust-Region Subproblem (GTRS):

$$
\min _{\mathbf{z} \in \Re^{r+1}}\|\bar{A} \mathbf{z}-\mathbf{c}\|^{2} \quad \text { s.t. } \mathbf{z}^{T} C \mathbf{z}+2 \mathbf{g}^{T} \mathbf{z}=0
$$

where $\mathbf{z}^{T}:=\left(\mathbf{x}^{T}, \rho_{0}\right)$ and

$$
\begin{gathered}
\bar{A}:=\left[\begin{array}{cc}
-2 \mathbf{a}_{1}^{T} & 1 \\
\vdots & \vdots \\
-2 \mathbf{a}_{m}^{T} & 1
\end{array}\right], \mathbf{c}:=\left[\begin{array}{c}
r_{1}^{2}-\left\|\mathbf{a}_{1}\right\|^{2} \\
\vdots \\
r_{m}^{2}-\left\|\mathbf{a}_{m}\right\|^{2}
\end{array}\right], \\
C:=\left[\begin{array}{cc}
I_{r} & 0_{r \times 1} \\
0_{1 \times r} & 0
\end{array}\right], \mathbf{g}:=\left[\begin{array}{c}
0_{1 \times r} \\
-0.5
\end{array}\right] .
\end{gathered}
$$

It follows from [21, Thm. 3.2] that $\mathbf{z} \in \Re^{r+1}$ is an optimal solution of (24) if and only if there exists $\rho \in \Re$ such that

$$
\left\{\begin{aligned}
\left(\bar{A}^{T} \bar{A}+\rho C\right) \mathbf{z} & =\bar{A}^{T} \mathbf{c}-\rho \mathbf{g}, \\
\mathbf{z}^{T} C \mathbf{z}+2 \mathbf{g}^{T} \mathbf{z} & =0 \\
\bar{A}^{T} \bar{A}+\rho C & \succeq 0 .
\end{aligned}\right.
$$

There are two assumptions that ensure an efficient solution algorithm of (24).

(H1) The matrix $\bar{A}$ defined in (25) has full column rank.

(H2) The matrix $\left(\bar{A}^{T} \bar{A}+\rho C\right)$ is positive definite, where $\rho$ satisfies (26).

Under the two assumptions, $\rho$ is the unique solution of

$$
\varphi(\rho)=0 \quad \text { and } \quad \bar{A}^{T} \bar{A}+\rho C \succ 0,
$$


where

$$
\varphi(\rho):=\hat{\mathbf{z}}(\rho)^{T} C \hat{\mathbf{z}}(\rho)+2 \mathbf{g}^{T} \hat{\mathbf{z}}(\rho)
$$

and

$$
\hat{\mathbf{z}}(\rho):=\left(\bar{A}^{T} \bar{A}+\rho C\right)^{-1}\left(\bar{A}^{T} \mathbf{c}-\rho \mathbf{g}\right) .
$$

Furthermore, there exists an efficient solution method to find the root of (27) (see [3] for details). It is emphasized in [3, P.1773] that the "hard case" (i.e., $\left(\bar{A}^{T} \bar{A}+\rho C\right)$ is just positive semidefinite) never occurred in their extensive numerical simulations.

We now state a result of implications from those two assumptions. Without loss of generality and for simplicity, we assume that the anchors $\left\{\mathbf{a}_{1}, \ldots, \mathbf{a}_{m}\right\}$ are centered at the origin. That is, $\sum_{i=1}^{m} \mathbf{a}_{i}=0$. Otherwise, we can always consider the points $\left\{\overline{\mathbf{a}}_{1}, \ldots, \overline{\mathbf{a}}_{m}\right\}$ instead of $\left\{\mathbf{a}_{1}, \ldots, \mathbf{a}_{m}\right\}$, where $\overline{\mathbf{a}}_{i}:=\mathbf{a}_{i}-\overline{\mathbf{a}}$ and $\overline{\mathbf{a}}:=\sum_{i=1}^{m} \mathbf{a}_{i} / m$.

Proposition 4.1: Suppose that there is another set of $m$ sensors in $\Re^{r}$, denoted by $\left\{\overline{\mathbf{z}}_{1}, \ldots, \overline{\mathbf{z}}_{m}\right\}$, satisfying

$$
\left\{\begin{array}{l}
\sum_{i=1}^{m} \mathbf{a}_{i}=\sum_{i=1}^{m} \overline{\mathbf{z}}_{i}=0,\left\|\mathbf{a}_{i}\right\|=\left\|\mathbf{z}_{i}\right\| \\
\left\|\overline{\mathbf{z}}_{i}-\overline{\mathbf{z}}_{j}\right\|^{2}=\left\|\mathbf{a}_{i}-\mathbf{a}_{j}\right\|^{2}, \forall i, j=1, \ldots, m .
\end{array}\right.
$$

Define

$$
Z_{1}^{T}:=\left[\overline{\mathbf{z}}_{1}, \ldots, \overline{\mathbf{z}}_{m}\right], A^{T}:=\left[\mathbf{a}_{1}, \ldots, \mathbf{a}_{m}\right] \text { and } P:=U V^{T},
$$

where $U$ and $V$ come from the singular value decomposition

$$
Z_{1}^{T} A=U \Sigma V^{T}
$$

with $\Sigma$ being the diagonal matrix of the singular values of $Z_{1}^{T} A$ and $U, V \in \mathcal{O}^{r}$. We have the following results.

(i) Assumption (H1) implies

$$
A=Z_{1} P .
$$

(ii) Let $\mathbf{x}$ denote an optimal solution of (1) and $\overline{\mathbf{z}}_{n}$ denote an optimal solution of (1) when the set of locations $\left\{\overline{\mathbf{z}}_{1}, \ldots, \overline{\mathbf{z}}_{m}\right\}$ are used instead of $\left\{\mathbf{a}_{1}, \ldots, \mathbf{a}_{m}\right\}$. Under Assumption (H2), we must have

$$
\overline{\mathbf{z}}_{n}=P \mathbf{x} .
$$

Proof: (i) Define the linear operator $\mathcal{L}: \mathcal{S}^{m} \mapsto \mathcal{S}^{m}$ by

$$
\mathcal{L}(X):=\operatorname{diag}(X) e^{T}+e \operatorname{diag}(X)^{T}-2 X, \quad \forall X \in \mathcal{S}^{n} .
$$

It follows that

$$
\begin{aligned}
\left(\mathcal{L}\left(Z_{1} Z_{1}^{T}\right)\right)_{i j} & =\left\|\overline{\mathbf{z}}_{i}\right\|^{2}+\left\|\overline{\mathbf{z}}_{j}\right\|^{2}-2 \overline{\mathbf{z}}_{i}^{T} \overline{\mathbf{z}}_{j} \\
& =\left\|\overline{\mathbf{z}}_{i}-\overline{\mathbf{z}}_{j}\right\|^{2} \\
& =\left\|\mathbf{a}_{i}-\mathbf{a}_{j}\right\|^{2} \\
& =\left(\mathcal{L}\left(A A^{T}\right)\right)_{i j} .
\end{aligned}
$$


The identity $\mathcal{L}\left(Z_{1} Z_{1}^{T}\right)=\mathcal{L}\left(A A^{T}\right)$ implies

$$
Z_{1} Z_{1}^{T}=A A^{T}
$$

The full column rank of $\bar{A}$ implies that $m \geq r+1$ and the affine hull of $\left\{\mathbf{a}_{1}, \ldots, \mathbf{a}_{m}\right\}$ spans $\Re^{r}$. It follows from [18, Thms. 5.1 and 5.2] that there exists an orthogonal matrix $P \in \mathcal{O}^{r}$ such that

$$
Z_{1} P=A \quad \text { and } \quad P=U V^{T}
$$

where $U$ and $V$ come from the singular value decomposition $Z_{1}^{T} A=U \Sigma V^{T}$ and $\Sigma$ is the diagonal matrix with the singular values of $Z_{1}^{T} A$ being its diagonal.

(ii) The optimal location of $\mathrm{x}$ is given by

$$
\hat{\mathbf{z}}(\rho)=\left[\begin{array}{l}
\mathbf{x} \\
\rho_{0}
\end{array}\right]=\left(\bar{A}^{T} \bar{A}+\rho C\right)^{-1}\left(\bar{A}^{T} \mathbf{c}-\rho \mathbf{g}\right) \quad \text { for some } \rho .
$$

Define

$$
\bar{Z}_{1}:=\left[\begin{array}{ll}
-2 Z_{1} & e
\end{array}\right] \text { and } \bar{P}:=\left[\begin{array}{cc}
P & 0_{1 \times r} \\
0_{r \times 1} & 1
\end{array}\right] .
$$

Then $\bar{P}$ is orthogonal and

$$
\bar{A}=\left[\begin{array}{ll}
-2 A & e
\end{array}\right]=\left[\begin{array}{ll}
-2 Z_{1} P & e
\end{array}\right]=\bar{Z}_{1} \bar{P}
$$

It is easy to calculate that

$$
\bar{P}^{T}\left(\bar{Z}_{1}^{T} \bar{Z}_{1}+\rho C\right) \bar{P}=\bar{A}^{T} \bar{A}+\rho C \succ 0 .
$$

Therefore, $\bar{Z}_{1}^{T} \bar{Z}_{1}+\rho C \succ 0$. Replacing $\bar{A}$ by $\bar{Z}_{1}$ in (26) will give rise to another set of necessary and sufficient conditions in terms of $Z_{1}$. Define

$$
\overline{\mathbf{z}}(\rho):=\left(\bar{Z}_{1}^{T} \bar{Z}_{1}+\rho C\right)^{-1}\left(\bar{Z}_{1}^{T} \mathbf{c}-\rho \mathbf{g}\right) .
$$

It follows from $\left(\bar{Z}_{1}^{T} \bar{Z}_{1}+\rho C\right)^{-1}=\bar{P}\left(\bar{A}^{T} \bar{A}+\rho C\right)^{-1} \bar{P}^{T}$ that

$$
\begin{aligned}
\overline{\mathbf{z}}(\rho) & =\bar{P}\left(\bar{A}^{T} \bar{A}+\rho C\right)^{-1} \bar{P}^{T}\left(\bar{Z}_{1}^{T} \mathbf{c}-\rho \mathbf{g}\right) \\
& =\bar{P}\left(\bar{A}^{T} \bar{A}+\rho C\right)^{-1}\left(\bar{A}^{T} \mathbf{c}-\rho \mathbf{g}\right) \\
& =\bar{P} \hat{\mathbf{z}}(\rho) .
\end{aligned}
$$

The second equality above used (31) and $\bar{P}^{T} \mathrm{~g}=\mathrm{g}$. Furthermore, the function

$$
\begin{aligned}
\bar{\varphi}(\rho) & :=\overline{\mathbf{z}}(\rho)^{T} C \overline{\mathbf{z}}(\rho)+2 \mathbf{g}^{T} \overline{\mathbf{z}}(\rho) \\
& =\hat{\mathbf{z}}(\rho)^{T} \bar{P}^{T} C \bar{P} \hat{\mathbf{z}}(\rho)+2 \mathbf{g}^{T} \bar{P} \hat{\mathbf{z}}(\rho) \\
& =\hat{\mathbf{z}}(\rho)^{T} C \hat{\mathbf{z}}(\rho)+2 \mathbf{g}^{T} \hat{\mathbf{z}}(\rho) \\
& =\varphi(\rho)=0 .
\end{aligned}
$$


The penultimate equality used $\bar{P}^{T} C \bar{P}=C$ and $\bar{P}^{T} \mathbf{g}=\mathrm{g}$. Therefore, $\overline{\mathbf{z}}(\rho)$ must be an optimal solution and its last element must be $\rho_{0}$. Denote the first $m$ elements of $\overline{\mathbf{z}}(\rho)$ by the vector $\overline{\mathbf{z}}_{n}$. It follows (32) that

$$
\overline{\mathbf{z}}(\rho)=\left[\begin{array}{c}
\overline{\mathbf{z}}_{n} \\
\rho_{0}
\end{array}\right]=\left[\begin{array}{c}
P \mathbf{x} \\
\rho_{0}
\end{array}\right],
$$

which gives $\overline{\mathbf{z}}_{n}=P \mathbf{x}$.

We have two remarks regrading this result.

(R1) Prop. 4.1 has a very nice geometrical interpretation. Let the $m$ known anchors be placed at two sets of locations, denoted by $\left\{\mathbf{a}_{1}, \ldots, \mathbf{a}_{m}\right\}$ and $\left\{\overline{\mathbf{z}}_{1}, \ldots, \overline{\mathbf{z}}_{m}\right\}$ respectively. Suppose they are centred and obey the pairwise distances in the sense of (28). Assumption (H1) means that one set of locations can be obtained from the other through an orthogonal rotation. As in the result, let $\mathbf{x}$ and $\overline{\mathbf{z}}_{n}$ denote the respective optimal solution of (1) when the two sets of $m$ anchors were used. Assumption (H2) implies that the recovered location $\mathbf{x}$ of the unknown source can be obtained through the same orthogonal rotation of $\overline{\mathbf{z}}_{n}$.

(R2) Without Assumption (H2) (i.e., $\bar{A}^{T} \bar{A}+\rho C$ is only positive semidefinite, which is referred to as the "hard case" in the literature of trust region methods), it is not known whether we would still have the same interpretation in (R1). The difficulty lies with the facts that there might be multiple solutions $\mathbf{x}$ of (1) and that it is very hard to characterize them (see [21, Sect. 5]).

\section{B. Equivalence to GTRS}

Let the optimal solution of (15) be denoted by $\bar{y}:=(\bar{u}, \bar{v}) \in \Re^{n} \times \Re^{m(m-1) / 2}$ (recall $n=m+1$ ). We assume that

$$
\bar{Y}=\Pi_{\mathcal{K}_{+}^{n}(r)}\left(-D+\mathcal{A}^{*}(\bar{y})\right)
$$

solves (9) (such $\bar{Y}$ exists, for example, under the conditions in Thm. 3.6).

The Schoenberg-Young-Householder theorem [27], [30] says (note that $(-\bar{Y})$ is a Euclidean distance matrix as we negated $Y$ in leading to (9)) that the rows of $Z \in \Re^{n \times r}$, which satisfies

$$
\frac{1}{2} J \bar{Y} J=Z Z^{T}
$$

are localizations of $(m+1)$ points that satisfy the constraints in (9). Let $\mathbf{z}_{i}$ denote the coordinates (column vector) of the $i$ th point and

$$
Z^{T}=\left[\mathbf{z}_{1}, \ldots, \mathbf{z}_{m+1}\right]
$$

Then we have

$$
-\bar{Y}_{i j}=\left\|\mathbf{z}_{i}-\mathbf{z}_{j}\right\|^{2}, \quad \forall i, j=1, \ldots, m+1
$$

and

$$
\left\|\mathbf{z}_{i}-\mathbf{z}_{j}\right\|^{2}=D_{i j}=\left\|\mathbf{a}_{i}-\mathbf{a}_{j}\right\|^{2}, \quad \forall i, j=1, \ldots, m .
$$


Define

$$
\mathbf{z}_{0}:=\sum_{i=1}^{m} \mathbf{z}_{i} / m \text { and } \overline{\mathbf{z}}_{i}:=\mathbf{z}_{i}-\mathbf{z}_{0}, \forall i=1, \ldots, m+1 .
$$

Then the set of locations of the first $m$ points $\left\{\overline{\mathbf{z}}_{1}, \ldots, \overline{\mathbf{z}}_{m}\right\}$ are centered at the origin and they obviously satisfy $\left\|\overline{\mathbf{z}}_{i}-\overline{\mathbf{z}}_{j}\right\|=\left\|\mathbf{a}_{i}-\mathbf{a}_{j}\right\|$ for $i, j=1, \ldots, m$. According to Prop. 4.1, there exists an orthogonal matrix $P \in \mathcal{O}^{r}$ such that

$$
\overline{\mathbf{z}}_{i}=P \mathbf{a}_{i}, \quad \forall i=1, \ldots, m \quad \text { and } \quad \overline{\mathbf{z}}_{m+1}=P \mathbf{x},
$$

where $\mathbf{x}$ is the optimal solution of (1). In other words, the locations of $\left(\mathbf{z}_{1}, \ldots, \mathbf{z}_{n}\right)$ can be translated to $\left(\mathbf{a}_{1}, \ldots, \mathbf{a}_{m}, \mathbf{x}\right)$ after the shifting (37) and rotation (38). We note that those localizations are also centered:

$$
Z^{T} e=\sum_{i=1}^{m+1} \mathbf{z}_{i}=0
$$

This can be easily verified through (34) as $J e=0$. We have the following technical lemma.

Lemma 4.2: Recall $\bar{y}:=(\bar{u}, \bar{v}) \in \Re^{m+1} \times \Re^{m(m-1) / 2}$ is the optimal solution of (15). We have

$$
\bar{u}_{m+1}=-\sum_{i=1}^{m}\left(\left\|\mathbf{a}_{i}-\mathbf{x}\right\|^{2}-r_{i}^{2}\right) .
$$

Proof: Throughout this proof, we use the fact $n=m+1$. Denote

$$
\left[\begin{array}{ll}
\widehat{Y}_{1} & \hat{y} \\
\hat{y}^{T} & \hat{y}_{0}
\end{array}\right]:=Q\left(-D+\mathcal{A}^{*}(\bar{y})\right) Q,
$$

where $\widehat{Y}_{1} \in \mathcal{S}^{m}$. It follows from (11) that

$$
\begin{aligned}
\bar{Y}= & \Pi_{\mathcal{S}_{+}^{n}(r)}\left(J\left(-D+\mathcal{A}^{*}(\bar{y})\right) J\right) \\
& +\left(-D+\mathcal{A}^{*}(\bar{y})\right)-\left(J\left(-D+\mathcal{A}^{*}(\bar{y})\right) J\right) \\
= & J \bar{Y} J+\left(-D+\mathcal{A}^{*}(\bar{y})\right)-\left(J\left(-D+\mathcal{A}^{*}(\bar{y})\right) J\right) \\
= & 2 Z Z^{T}+\left(-D+\mathcal{A}^{*}(\bar{y})\right)-Q\left[\begin{array}{cc}
\widehat{Y}_{1} & 0 \\
0 & 0
\end{array}\right] Q .
\end{aligned}
$$

The second equality above used (13) and the last equality used (34) and (20). Because $(-\bar{Y})$ is the EDM generated by $\left\{\mathbf{z}_{1}, \ldots, \mathbf{z}_{n}\right\}$, the last column of $\bar{Y}$ is

$$
\bar{Y}(:, n)=-\left[\left\|\mathbf{z}_{1}-\mathbf{z}_{n}\right\|^{2}, \ldots,\left\|\mathbf{z}_{m}-\mathbf{z}_{n}\right\|^{2}, 0\right]^{T} .
$$

We compare the last column of $\bar{Y}$ with the last column of the right-hand-side matrix in (40).

$$
\begin{aligned}
-\left[\begin{array}{c}
\left\|\mathbf{z}_{1}-\mathbf{z}_{n}\right\|^{2} \\
\vdots \\
\left\|\mathbf{z}_{m}-\mathbf{z}_{n}\right\|^{2} \\
0
\end{array}\right]=2\left[\begin{array}{c}
\left\langle\mathbf{z}_{1}, \mathbf{z}_{n}\right\rangle \\
\vdots \\
\left\langle\mathbf{z}_{m}, \mathbf{z}_{n}\right\rangle \\
\left\langle\mathbf{z}_{n}, \mathbf{z}_{n}\right\rangle
\end{array}\right]+\left[\begin{array}{c}
-r_{1}^{2} \\
\vdots \\
-r_{m}^{2} \\
\bar{u}_{m+1}
\end{array}\right] \\
-Q\left[\begin{array}{cc}
\widehat{Y}_{1} & 0 \\
0 & 0
\end{array}\right] Q e_{n} .
\end{aligned}
$$


Here, we use the structure of $\mathcal{A}^{*}(\bar{y})$, whose last column is $\left[0, \cdots, 0, \bar{u}_{m+1}\right]^{T}$. And $e_{n}$ is the $n$th coordinate vector in $\Re^{n}$. By the definition of $Q$, we have $Q e_{n}=-e / \sqrt{n}$ and

$$
\begin{gathered}
Q\left[\begin{array}{cc}
\widehat{Y}_{1} & 0 \\
0 & 0
\end{array}\right] Q e_{n}=-\frac{1}{\sqrt{n}} Q\left[\begin{array}{c}
\widehat{Y}_{1} e \\
0
\end{array}\right] \\
=\left[\begin{array}{c}
-\frac{1}{\sqrt{n}} \widehat{Y}_{1} e+\frac{e^{T} \widehat{Y}_{1} e}{\sqrt{n}(n+\sqrt{n})} e \\
0
\end{array}\right]+\left[\begin{array}{c}
0 \\
e^{T} \widehat{Y}_{1} e / n
\end{array}\right] .
\end{gathered}
$$

Therefore, we have

$$
\begin{aligned}
-\left[\begin{array}{c}
\left\|\mathbf{z}_{1}-\mathbf{z}_{n}\right\|^{2} \\
\vdots \\
\left\|\mathbf{z}_{m}-\mathbf{z}_{n}\right\|^{2} \\
0
\end{array}\right]=2\left[\begin{array}{c}
\left\langle\mathbf{z}_{1}, \mathbf{z}_{n}\right\rangle \\
\vdots \\
\left\langle\mathbf{z}_{m}, \mathbf{z}_{n}\right\rangle \\
\left\langle\mathbf{z}_{n}, \mathbf{z}_{n}\right\rangle
\end{array}\right]+\left[\begin{array}{c}
-r_{1}^{2} \\
\vdots \\
-r_{m}^{2} \\
\bar{u}_{m+1}
\end{array}\right] \\
-\left[\begin{array}{c}
-\frac{1}{\sqrt{n}} \widehat{Y}_{1} e+\frac{e^{T} \widehat{Y}_{1} e}{\sqrt{n}(n+\sqrt{n})} e \\
e^{T} \widehat{Y}_{1} e / n
\end{array}\right]
\end{aligned}
$$

The last equation in (41) gives

$$
\bar{u}_{m+1}=e^{T} \widehat{Y}_{1} e / n-2\left\|\mathbf{z}_{n}\right\|^{2}
$$

Summing the first $m$ equations in (41) yields

$$
\begin{aligned}
- & \sum_{i=1}^{m}\left\|\mathbf{z}_{i}-\mathbf{z}_{n}\right\|^{2}=2 \sum_{i=1}^{m}\left\langle\mathbf{z}_{i}, \mathbf{z}_{n}\right\rangle- \\
& \sum_{i=1}^{n} r_{i}^{2}+\frac{1}{\sqrt{n}} e^{T} \widehat{Y}_{1} e-\frac{n-1}{\sqrt{n}(n+\sqrt{n})} e^{T} \widehat{Y}_{1} e \\
= & 2 \sum_{i=1}^{m}\left\langle\mathbf{z}_{i}, \mathbf{z}_{n}\right\rangle-\sum_{i=1}^{n} r_{i}^{2}+e^{T} \widehat{Y}_{1} e / n .
\end{aligned}
$$

Subtracting (43) from (42) and re-arranging the like-terms leads to

$$
\begin{array}{rlr}
\bar{u}_{m+1} & =-\sum_{i=1}^{m}\left(\left\|\mathbf{z}_{i}-\mathbf{z}_{n}\right\|^{2}-r_{i}^{2}\right)-2\left\langle\sum_{i=1}^{m} \mathbf{z}_{i}+\mathbf{z}_{n}, \mathbf{z}_{n}\right\rangle \\
& =-\sum_{i=1}^{m}\left(\left\|\mathbf{z}_{i}-\mathbf{z}_{n}\right\|^{2}-r_{i}^{2}\right) & \text { (by (39)) } \\
& =-\sum_{i=1}^{m}\left(\left\|P\left(\mathbf{a}_{i}-\mathbf{x}\right)\right\|^{2}-r_{i}^{2}\right) & \text { (by (38)) } \\
& =-\sum_{i=1}^{m}\left(\left\|\mathbf{a}_{i}-\mathbf{x}\right\|^{2}-r_{i}^{2}\right) .
\end{array}
$$

This proves our result.

Now we are ready to present our characterization of the solution of optimality condition (26). In the characterization, we list all the assumptions that have been made so far to clarify the situation when such characterization holds. 
The first two assumptions, as assumed in [3], remove the possibility of "hard case" of the trust region subproblem from happening and the third condition assumes that the problem (9) can be solved globally. The result is that there is a close relationship between the obtained solutions, as characterized below.

Theorem 4.3: Consider the SSLP problem (1). Let the matrices $\bar{A}$ and $C$ be defined as in (25). Let $\bar{y}:=(\bar{u}, \bar{v}) \in$ $\Re^{m+1} \times \Re^{m(m-1) / 2}$ solve the dual problem (15). Assume that Assumptions (H1) and (H2) hold and that the matrix $\bar{Y}$ defined in (33) is an optimal solution of (9). Then, we must have

$$
\rho=-2 \bar{u}_{m+1},
$$

where $\rho$ is the (unique) optimal solution of (26). Moreover, the two problems (1) and (9) return the same optimal objective value.

Proof: Consider the optimality condition (26). The second equation in (26) simply requires $\rho_{0}=\|\mathbf{x}\|^{2}$. It is easy to see that

$$
\bar{A} \mathbf{z}=-2\left[\begin{array}{c}
\left\langle\mathbf{a}_{1}, \mathbf{x}\right\rangle \\
\vdots \\
\left\langle\mathbf{a}_{m}, \mathbf{x}\right\rangle
\end{array}\right]+\rho_{0} e, \quad C \mathbf{z}=\left[\begin{array}{c}
\mathbf{x} \\
0
\end{array}\right]
$$

and

$$
\mathbf{c}=\left[\begin{array}{c}
r_{1}^{2} \\
\vdots \\
r_{m}^{2}
\end{array}\right]-\left[\begin{array}{c}
\left\|\mathbf{a}_{1}\right\|^{2} \\
\vdots \\
\left\|\mathbf{a}_{m}\right\|^{2}
\end{array}\right]=: \mathbf{p}_{1}-\mathbf{p}_{2}
$$

The first equation in (26) can be written as

$$
\bar{A}^{T}\left(\bar{A} \mathbf{z}+\mathbf{p}_{2}\right)+\rho\left[\begin{array}{l}
\mathbf{x} \\
0
\end{array}\right]=\bar{A}^{T} \mathbf{p}_{1}-\rho \mathbf{g} .
$$

We note that the $i$ th component of $\left(A \mathbf{z}+\mathbf{p}_{2}\right)$ is $\left\|\mathbf{a}_{i}-\mathbf{x}\right\|^{2}$. After re-arranging terms in (44), we obtain

$$
\bar{A}^{T}\left[\begin{array}{c}
\left\|\mathbf{a}_{1}-\mathbf{x}\right\|^{2}-r_{1}^{2} \\
\vdots \\
\left\|\mathbf{a}_{m}-\mathbf{x}\right\|^{2}-r_{m}^{2}
\end{array}\right]=-\rho\left[\begin{array}{c}
0 \\
-0.5
\end{array}\right]-\rho\left[\begin{array}{l}
\mathbf{x} \\
0
\end{array}\right],
$$

which by the definition of $\bar{A}$ yields

$$
\rho=2 \sum_{i=1}^{m}\left(\left\|\mathbf{a}_{i}-\mathbf{x}\right\|^{2}-r_{i}^{2}\right)=-2 \bar{u}_{m+1} . \quad \text { (by Lemma 4.2) }
$$


Now let $\left\{\mathbf{z}_{i}\right\}$ be defined by (37). The optimal objective value of (9) is

$$
\begin{aligned}
& \frac{1}{2}\|\bar{Y}+D\|^{2} \\
= & \frac{1}{2} \sum_{i, j=1}^{m}\left(\bar{Y}_{i j}+D_{i j}\right)^{2}+\sum_{i=1}^{m}\left(\bar{Y}_{i(m+1)}+D_{i(m+1)}\right)^{2} \\
= & \frac{1}{2} \sum_{i, j=1}^{m}\left(\left\|\mathbf{z}_{i}-\mathbf{z}_{j}\right\|^{2}-D_{i j}\right)^{2}+ \\
& \sum_{i=1}^{m}\left(\left\|\mathbf{z}_{i}-\mathbf{z}_{m+1}\right\|^{2}-D_{i(m+1)}\right)^{2} \quad \text { (by (35)) } \\
= & \sum_{i=1}^{m}\left(\left\|\mathbf{z}_{i}-\mathbf{z}_{m+1}\right\|^{2}-D_{i(m+1)}\right)^{2} \quad \text { (by (36)) } \\
= & \sum_{i=1}^{m}\left(\left\|\overline{\mathbf{z}}_{i}-\overline{\mathbf{z}}_{m+1}\right\|^{2}-r_{i}^{2}\right)^{2} \quad \text { (by (37)) } \\
= & \sum_{i=1}^{m}\left(\left\|P \mathbf{a}_{i}-P \mathbf{x}\right\|^{2}-r_{i}^{2}\right)^{2} \quad \text { (by (38)) } \\
= & \sum_{i=1}^{m}\left(\left\|\mathbf{a}_{i}-\mathbf{x}\right\|^{2}-r_{i}^{2}\right)^{2} . \quad
\end{aligned}
$$

Hence, the two problems (1) and (9) return the same optimal objective value.

This result characterizes the nice situation defined by the assumptions (H1) and (H2). The unpleasant situation happens when (H1) and (H2) fail to hold. As already remarked in (R2) after Prop. 4.1, it would be hard to characterize the optimal solution of the GTRS. The good thing is that the failure of (H1) or (H2) does not contradict the zeroduality gap result in Thm. 3.6. In other words, the Lagrangian dual approach would still have chance to find the global solution of the SSLP under this situation.

\section{NUMERICAL EXAMPLES}

This numerical part includes three subsections. Subsect. V-A uses the existing SSLP test problems to show that the Lagrangian Dual (denoted as LagD) method provides localization of similar quality as that by the GTRS. Subsect. V-B further explores the possibility of including more constraints in the SSLP model. For example, if two more fixed constraints of the type (45) are added to the model, LagD can accurately recover the unknown source $\mathbf{x}=(-2,3)$ in Example 5.1. In theory, the Lagrangian dual approach can be straightforwardly extended to the problem of multiple source localizations. Subsect. V-C demonstrates its efficiency on three standard test problems (e.g., the number of unknown sources are up to 300) by comparison with the popular SDP solver SNLSDP [5] ${ }^{1}$. All of our results can be reproduced by using the Matlab code LagD ${ }^{2}$. All tests were carried out using the 64-bit version of MATLAB 2012a on a Windows 7 desktop with a 64-bit operating system having Intel Core 2 Duo CPU of $3.16 \mathrm{GHz}$ and 4.0GB of RAM.

\footnotetext{
${ }^{1}$ Available from http://www.math.nus.edu.sg/ mattohkc/SNLSDP.html.

${ }^{2}$ Available from http://www.personal.soton.ac.uk/hdqi.
} 


\section{A. The Case of Single Source}

The single source localization problem, treated as a Least-Square (LS) problem in [3], [4], can be solved by reformulating it as a generalized trust-region subproblem (GTRS). The Lagrangian dual approach, as proved in Thm. 4.3 for this case, also solves the GTRS under the conditions assumed in [3]. We use the two examples tested in [3], [4] to verify this theoretical result. We used HANSO package to solve the Lagrangian dual problem (15).

Example 5.1: [3, Example 1] There are 5 known sensors (anchors) $\left\{\mathbf{a}_{i}\right\}$ in the plane $(r=2)$ whose coordinates are

$$
\left\{(6,4)^{T},(0,-10)^{T},(5,-3)^{T},(1,-4)^{T},(3,-3)^{T}\right\} .
$$

The unknown source's coordinates are $\mathbf{x}=(-2,3)^{T}$. The exact distances $\left(\left\|\mathbf{x}-\mathbf{a}_{i}\right\|\right)$ and the observed noisy distances $\left(r_{i}=\left\|\mathbf{x}-\mathbf{a}_{i}\right\|+\epsilon_{i}\right)$ are given by

$$
\begin{array}{llllll}
\text { exact } & 8.0622 & 13.1529 & 9.2195 & 7.6157 & 7.8102 \\
\text { noisy } & 8.0051 & 13.0112 & 9.1138 & 7.7924 & 8.0210 .
\end{array}
$$

Each noise component $\epsilon_{i}$ is a realization of a Gaussian distributed random variable with mean zero and standard deviation 0.1 . The GTRS solution is $(-2.018,2.9585)$, which is a relatively good approximation of the true source location. The solution obtained by the Lagrangian dual approach is $(-2.0189,2.9585)$, same as obtained by GTRS. The Lagrange multiplier $\bar{u}_{m+1}=1.1122$. Therefore, $\rho=-2 \bar{u}_{m+1}$ should be a good approximation to the optimal solution of GTRS by Thm. 4.3.

Example 5.2: [4, Example 4.3] In this example, 100 instances are randomly generated. In each instance, there are 5 sensors whose locations $\mathbf{a}_{j}$ and the source location $\mathbf{x}$ are randomly generated from a uniform distribution over the square $[-10,10] \times[-10,10]$. The observed distances $d_{j}$ are given by $\left\|\mathbf{a}_{j}-\mathbf{x}\right\|+\epsilon_{j}$ with $\epsilon_{j}$ being independently generated from a normal distribution with mean zero and standard deviation $\sigma$.

We tested GTRS and the Lagrangian dual method LagD on Example 5.2. GTRS aims at finding the root of $\varphi(\rho)=0$ in (27). It is known that the root is contained in the interval $(\underline{\rho}, \infty)$ and $\varphi(\rho)$ is strictly decreasing over this interval, where

$$
\underline{\rho}=-1 / \lambda_{1}\left(C, \bar{A}^{T} \bar{A}\right)
$$

and $\lambda_{1}\left(C, \bar{A}^{T} \bar{A}\right)$ is the largest eigenvalue of the matrix $\left(\bar{A}^{T} \bar{A}\right)^{-1 / 2} C\left(\bar{A}^{T} \bar{A}\right)^{-1 / 2}$ (see [3, Sect. II(B)]).

In our implementation of GTRS, we first narrow the half-line interval $(\underline{\rho}, \infty)$ to $(\underline{\rho}, \bar{\rho})$, where $\bar{\rho}=\underline{\rho}+10 i_{0}$ and $i_{0}$ is the first positive integer $i$ such that $\varphi(\underline{\rho}+10 i)<0$. By the strictly decreasing property of $\varphi$, the root must lie in the interval $(\underline{\rho}, \bar{\rho})$. We then use the bisection method (as suggested in [3]) to find the root. The tolerance used to terminate GTRS is $|\varphi(\rho)| \leq 10^{-3}$ (smaller tolerance such as $10^{-5}$ would not achieve significantly more accuracy in localization). For GTRS, Iter is the number of function evaluations of $\varphi$ till the termination. For LagD, Iter is the number of iterations used by HANSO package. We also report the squared position error $\|\overline{\mathbf{x}}-\mathbf{x}\|^{2}$, where $\overline{\mathbf{x}}$ is the solution by the corresponding method. All results are based on the average over 100 random realizations. It can be seen that LagD and GTRS return localizations with highly similar squared position errors, which are consistent with our theoretical finding in Theorem 4.3. 
TABLE I

MEAN SQUARED POSITION ERROR OF GTRS AND LAgD METHODS

\begin{tabular}{|c|cr|cr|}
\hline Example 5.2 & \multicolumn{2}{|c|}{ GTRS } & \multicolumn{2}{c|}{ LagD } \\
\hline$\sigma$ & Iter & $\|\overline{\mathbf{x}}-\mathbf{x}\|^{2}$ & Iter & $\|\overline{\mathbf{x}}-\mathbf{x}\|^{2}$ \\
\hline $1.00 \mathrm{E}-03$ & 11 & $1.20 \mathrm{E}-06$ & 36 & $1.21 \mathrm{E}-06$ \\
$1.00 \mathrm{E}-02$ & 11 & $1.38 \mathrm{E}-04$ & 41 & $1.38 \mathrm{E}-04$ \\
$1.00 \mathrm{E}-01$ & 12 & $1.73 \mathrm{E}-02$ & 39 & $1.73 \mathrm{E}-02$ \\
$1.00 \mathrm{E}+00$ & 42 & 1.46 & 37 & 1.45 \\
\hline
\end{tabular}

\section{B. Including More Constraints}

One advantage of the Lagrangian dual approach is its capability of incorporating more information about the unknown source. For example, we may have a good chance to know in advance the distance to the anchor $\mathbf{a}_{i}$ from the source $\mathbf{x}$, i.e.,

$$
\left\|\mathbf{x}-\mathbf{a}_{i}\right\|=r_{i} \quad \text { for some } i \text {. }
$$

Such constraints are common in the literature of Euclidean distance matrix completion problems in the form of that the distance $D_{i j}$ is known for some pair $(i, j)$. For example, such constraints appear in the Euclidean distance matrix completion formulation of the famous Kissing number problem of sphere packing (see [10, Problem 504]). Each of such prior information would introduce one more quadratic constraint to the GTRS approach, which would need one more Lagrangian multiplier $\rho$ in order to state the corresponding conditions of (26). The existing global optimality theory as well as the GTRS algorithm (i.e., bisection method) for the one quadratic equation case would run into difficulties in extension. In contrast, the Lagrangian dual approach would just take such a constraint as a linear equation because we always have (from Part II(c))

$$
r_{i}^{2}=\left\|\mathbf{x}-\mathbf{a}_{i}\right\|^{2}=Y_{i n}=0.5\left\langle E_{i n}, Y\right\rangle
$$

where $E_{i j}$ is the zero matrix except its having the value 1 at the positions $(i, j)$ and $(j, i)$.

In this case, the problem (9) then becomes

$$
\min \frac{1}{2}\|Y+D\|^{2} \text { s.t. }\left\{\begin{array}{l}
\mathcal{A}(Y)=b, Y \in \mathcal{K}_{+}^{n}(r) \\
\left\langle E_{i n}, Y\right\rangle=2 r_{i}^{2}
\end{array}\right.
$$

We can derive its corresponding Lagrangian dual problem, which is also unconstrained. Consequently, it would be straightforward to generalize the Lagrangian dual approach to including this case. In order to appreciate this capability of the Lagrangian dual approach, let us have another look at Example 5.1.

Example 5.3: Assume that the distance between $\mathbf{x}$ and $\mathbf{a}_{2}$ is known. That is, $\left\|\mathbf{x}-\mathbf{a}_{2}\right\|=\sqrt{173}$ (note that it is the largest distance among all measured distances). The Lagrangian dual approach found $\mathbf{x}=(-1.9982,3.0003)$, a much accurate localization with the error $\|\mathbf{x}-(-2,3)\|=1.8 \times 10^{-3}$. If the distances to the first two anchors 
$\mathbf{a}_{1}$ and $\mathbf{a}_{2}$ are also respectively known to be $\sqrt{65}$ and $\sqrt{173}$, the Lagrangian dual approach would recover the true $\mathrm{x}$ with the error $2.5 \times 10^{-8}$. The latter case would enforce two more quadratic constraints to the GTRS approach.

\section{The Case of Multiple Sources}

Another advantage of the Lagrangian dual approach is that the Euclidean distance embedding reformulation (7) of the SSLP does not depend on the number of the unknown source as long as we can define the initial matrix $D$. Therefore, it can handle multiple unknown sources. Below, we demonstrate its efficiency by comparison with the well known SDP relaxation method studied in [5] (denoted by SNLSDP) on several standard test problems.

Example 5.4: This is the test problem in [5, III(C)]. The true positions of the $n$ unknown sensors $\mathbf{x}_{1}, \ldots, \mathbf{x}_{n}$ are independently generated by the uniform distribution on the unit square $[-0.5,0.5]^{2}$. The edge set $\mathcal{N}$ is generated by considering only pairs of points that have distances less than $R=0.3$ :

$$
\mathcal{N}=\left\{(i, j):\left\|\mathbf{x}_{i}-\mathbf{x}_{j}\right\| \leq R, 1 \leq i<j \leq n\right\} .
$$

There are four ( $m=4$ ) anchors either taking to be (a) outer anchors $[ \pm 0.45, \pm 0.45]$ or (b) inner anchors $[ \pm 0.2, \pm 0.2]$. The edge set $\mathcal{M}$ is defined by

$$
\mathcal{M}=\left\{(i, k):\left\|\mathbf{x}_{i}-\mathbf{a}_{k}\right\| \leq R, 1 \leq i \leq n, 1 \leq k \leq m\right\} .
$$

It is assumed that the distances $d_{i j}$ and $d_{i k}$ are perturbed by random noises $\epsilon_{i j}$ and $\epsilon_{i k}$ as follows:

$$
\begin{aligned}
& d_{i j}=\left\|\mathbf{x}_{i}-\mathbf{x}_{j}\right\|\left|1+\mathrm{nf} * \epsilon_{i j}\right| \quad(i, j) \in \mathcal{N} \\
& d_{i k}=\left\|\mathbf{x}_{i}-\mathbf{a}_{k}\right\|\left|1+\operatorname{nf} * \epsilon_{i j}\right| \quad(i, k) \in \mathcal{M},
\end{aligned}
$$

where $\epsilon_{i j}, \epsilon_{i k}$ are independent standard normal random variables and $\mathrm{nf}$ is the noisy factor taken to be $\mathrm{nf}=0.2$.

Example 5.5: This is the test problem in [29] and is a modification of Example 5.4 in the following way. A total of $N$ sensors are generated as in Example 5.4. Set $m=0.1 N$ (i.e., $10 \%$ of the sensors are anchors) and $n=N-m$ are unknown sensors. The remaining calculations are the same as in Example 5.4.

To follow the SNLSDP solver [5] and also for the comparison purpose, we calculate the Root Mean Square Distance (RMSD) to measure the accuracy of the estimated positions $\hat{\mathbf{x}}_{i}, i=1, \ldots, n$ :

$$
\operatorname{RMSD}=\frac{1}{\sqrt{n}}\left(\sum_{i=1}^{n}\left\|\hat{\mathbf{x}}_{i}-\mathbf{x}_{i}\right\|^{2}\right)^{1 / 2} .
$$

One of the very useful techniques that have been developed in [5] to improve the localization quality of the SNLSDP solver is the Refinement step, which is a gradient descent method applied to the objective distance function defined in [5, Eq.(18)] (for details, see [5, Part V]). We found that this Refinement step also improves the localization quality of our LagD solver. Figure 1 shows the improvement of the localization of LagD before and after the Refinement step. Hence, we will report the resulting RMSD by LagD with this refinement step.

In Tables II and III, cpu time are in seconds. Iter denotes the number of iterations used. For LagD, it is the number of iterations used by HANSO. The initial point for HANSO is provided by the Newton method [23] on the 

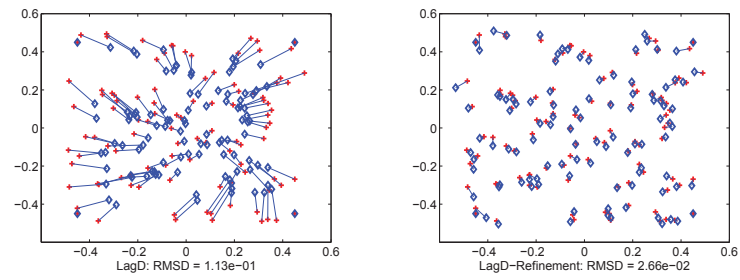

Fig. 1. Comparison of localizations of LagD before and after the Refinement step on Example 5.4: $n=100,4$ outer anchors, $R=0.3$, $\mathrm{nf}=0.2$, random state 0 . The symbol + represents the true positions of the sensors and $\diamond$ represents the localization by LagD.

convex relaxation problem of (7) with $\mathcal{K}_{+}^{n}(r)$ being replaced by its convex counterpart $\mathcal{K}_{+}^{n}$. All other parameters of HANSO are taken to be their default values.

We include here a brief discussion on the computational complexity of the algorithms involved. The complexity of LagD comes from two parts. One is computing the initial point by solving the convex relaxation problem of (7), which is done by the quadratically convergent Newton's method of [23]. The other part is from the HANSO package. We refer to [19] for its computational analysis (we report the number of iterations used by HANSO in Tables II and III). As for the GTRS, it is also an iterative algorithm and its complexity relies on how the function $\varphi(\rho)$ is computed or approximated. We refer to [3] for details. The SNLSDP solver solves a convex SDP and hence has polynomial time complexity, which does not necessarily imply it is faster (see Tables II and III).

We report the average results on 50 randomly generated instances for each of the examples. The rmsd columns in Tables II and III show that both LagD and SNLSDP yielded the localizations of similar quality in terms of rmsd (i.e., all in the order of $10^{-2}$ ). However, the cpu used by LagD is only a small fraction of that used by SNLSDP, especially when $n$ is of a few hundreds. Those results clearly demonstrate the potential of the Lagrangian dual approach for various localization problems. The research serves a good starting point for further investigations to be discussed in the next section.

Finally, we would like to point out that LagD used longer time for Example 5.4(a) ( $n=60)$ than that for $n=80,100$ (see Table II). This was due to the mechanism of HANSO package in evaluating the 'smallest" vector in the convex hull of the used gradients. Over the 50 random runs, there occurred a few cases that LagD used more iterations and hence longer time to terminate for $n=60$ compared to that for $n=80$ and $n=100$. This is reflected in the number of iterations used. In the table, Iter $=35$ for $n=60$ and Iter $=17$ and 20 for $n=80,100$ respectively.

\section{CONCLUSION}

There exist a few methods for source localization problems, especially for sensor network localization. For the single source localization problem, two methods stand out because of their global optimality properties. One is the generalized trust-region subproblem approach studied by Beck et al. [3]. Another is the Lagrangian dual approach studied by Qi [23] and Qi and Yuan [24] for Euclidean distance embedding problems. When specialized to the 
TABLE II

COMPARISON OF LAGD AND SNLSDP ON EXAMPLE 5.4(a): OUTER ANCHORS AND EXAMPLE 5.4(b): INNER ANCHORS.

\begin{tabular}{|c|c|c|c|c|c|c|c|}
\hline \multicolumn{2}{|c|}{ Examples } & \multicolumn{3}{|c|}{ LagD } & \multicolumn{3}{|c|}{ SNLSDP } \\
\hline Name & $n$ & срu & Iter & rmsd & сри & Iter & rmsd \\
\hline \multirow{8}{*}{ 5.4(a) } & 60 & 0.6 & 35 & $5.12 \mathrm{E}-2$ & 1.6 & 21 & $8.28 \mathrm{E}-2$ \\
\hline & 80 & 0.4 & 17 & $3.45 \mathrm{E}-2$ & 2 & 22 & $3.82 \mathrm{E}-2$ \\
\hline & 100 & 0.5 & 20 & 2.95E-2 & 4 & 23 & 2.93E-2 \\
\hline & 150 & 1 & 22 & $2.22 \mathrm{E}-2$ & 18 & 24 & 2.24E-2 \\
\hline & 180 & 2 & 23 & $1.89 \mathrm{E}-2$ & 39 & 24 & $1.91 \mathrm{E}-2$ \\
\hline & 200 & 3 & 24 & $1.84 \mathrm{E}-2$ & 61 & 23 & $1.87 \mathrm{E}-2$ \\
\hline & 250 & 5 & 26 & $1.65 \mathrm{E}-2$ & 182 & 23 & $1.67 \mathrm{E}-2$ \\
\hline & 300 & 10 & 29 & $1.44 \mathrm{E}-2$ & 494 & 23 & $1.46 \mathrm{E}-2$ \\
\hline \multirow{8}{*}{ 5.4(b) } & 60 & 0.3 & 16 & $4.49 \mathrm{E}-2$ & 1 & 21 & $9.86 \mathrm{E}-2$ \\
\hline & 80 & 0.3 & 16 & $3.25 \mathrm{E}-2$ & 2 & 22 & $3.45 \mathrm{E}-2$ \\
\hline & 100 & 0.5 & 15 & $2.86 \mathrm{E}-2$ & 4 & 22 & $2.78 \mathrm{E}-2$ \\
\hline & 150 & 1 & 15 & $1.98 \mathrm{E}-2$ & 20 & 24 & $1.98 \mathrm{E}-2$ \\
\hline & 180 & 1 & 16 & $1.83 \mathrm{E}-2$ & 43 & 23 & $1.83 \mathrm{E}-2$ \\
\hline & 200 & 3 & 16 & $1.68 \mathrm{E}-2$ & 68 & 23 & $1.66 \mathrm{E}-2$ \\
\hline & 250 & 5 & 16 & $1.49 \mathrm{E}-2$ & 196 & 23 & $1.48 \mathrm{E}-2$ \\
\hline & 300 & 10 & 17 & $1.37 \mathrm{E}-2$ & 563 & 23 & $1.36 \mathrm{E}-2$ \\
\hline
\end{tabular}

TABLE III

COMPARISON OF LAGD AND SNLSDP ON EXAMPLE 5.5: 10\% OF RANDOMLY GENERATED SENSORS ARE ANCHORS.

\begin{tabular}{|l|l|rrr|rll|}
\hline \multicolumn{2}{|c|}{ Examples } & \multicolumn{4}{c|}{ LagD } & \multicolumn{3}{c|}{ SNLSDP } \\
\hline \multirow{5}{*}{ Name } & $N$ & cpu & Iter & rmsd & cpu & Iter & rmsd \\
\hline \multirow{5}{*}{5.5} & 60 & 0.3 & 22 & $4.64 \mathrm{E}-2$ & 1 & 20 & $1.34 \mathrm{E}-1$ \\
& 10 & 0.3 & 18 & $3.18 \mathrm{E}-2$ & 2 & 22 & $4.09 \mathrm{E}-2$ \\
& 100 & 0.5 & 19 & $2.50 \mathrm{E}-2$ & 4 & 22 & $2.49 \mathrm{E}-2$ \\
& 180 & 1 & 26 & $1.87 \mathrm{E}-2$ & 18 & 23 & $1.83 \mathrm{E}-2$ \\
& 200 & 2 & 30 & $1.61 \mathrm{E}-2$ & 41 & 23 & $1.61 \mathrm{E}-2$ \\
& 250 & 5 & 38 & $1.32 \mathrm{E}-2$ & 199 & 23 & $1.31 \mathrm{E}-2$ \\
& 300 & 8 & 44 & $1.16 \mathrm{E}-2$ & 475 & 23 & $1.16 \mathrm{E}-2$ \\
\hline
\end{tabular}


SSLP, the Lagrangian dual approach is proved to be equivalent to the GTRS approach. Moreover, a nice geometrical interpretation of the assumptions used by the GTRS approach is revealed. We also study when the Lagrangian dual problem has an optimal solution and characterized when the zero duality gap holds for the SSLP.

The reported numerical results on SSLP are consistent with our theory in the sense that the resulting algorithm LagD provides localizations of similar quality as that of GTRS. Moreover, the numerical results on multiple source localization problems clearly shows the great potential of the Lagrangian dual approach when compared to the popular SNLSDP solver [5]. We also show that the Lagrangian dual approach can handle the fixed distance constraint of the type (45). A more general type of the distance constraint takes the form:

$$
\ell_{i j} \leq\left\|\mathbf{x}_{i}-\mathbf{x}_{j}\right\| \leq u_{i j} \quad \text { for some }(i, j)
$$

where $\ell_{i j}$ and $u_{i j}$ are the lower and upper bounds of the distance between $\mathbf{x}_{i}$ and $\mathbf{x}_{j}$. Such constraints arise from many real applications, see e.g., [15]. However, the current form of the Lagrangian dual approach cannot be directly used to deal with such constraints. We leave this as a future research topic.

Another future topic, as suggested by one referee, is to design efficient algorithms for the maximum likelihood (ML) formulation of the localization problem based on the efficient methods such as the Lagrangian dual approach for the least-squares (LS) formulation. A pioneering work has been done in [4], [2] which makes use of the GTRS to solve the ML formulation of the SSLP. We may follow a similar framework of [4] to design a sequential algorithm that uses the Lagrangian dual approach in each step.

\section{ACKNOWLEDGMENT}

We would like to thank the three referees as well as the associate editor for their constructive suggestions, which have significantly improved the quality of the paper.

\section{REFERENCES}

[1] A. Y. Alfakih, A. Khandani, and H. Wolkowicz, Solving Euclidean distance matrix completion problems via semidefinite programming, Comput. Optim. Appl., 12 (1999), pp. 13-30.

[2] A. Beck and D. Pan, On the solution of the GPS localization and circle fitting problems, SIAM. J. Optim., 22 (2012), pp. $108-134$.

[3] A. Beck, P. Stoica, and J. Li, Exact and approximate solutions of source localization problems, IEEE Tran. Sign. Proces., 56 (2008), pp. 1770-1778.

[4] A. Beck, M. Teboulle, and Z. Chikishev, Iterative minimization schemes for solving the single source localization problem, SIAM J. Optim., 19 (2008), pp. 1397-1416.

[5] P. Biswas, T.-C. Lian, K.-C. Toh, T.-C. Wang, and Y. Ye, Semidefinite programming based algorithms for sensor network localization, ACM Trans. Sensor Networks 2 (2006), pp. 188-220.

[6] I. Borg and P. J. F. Groenen, Modern Multidimensional Scaling: Theory and Applications (2nd ed.) Springer Series in Statistics, Springer, 2005.

[7] K. W. Cheung, H. C. So, W. K. Ma, and Y. T. Chan, Least squares algorithms for time-of-arrival-based mobile location, IEEE Trans. Sign. Proces., 52 (2004), pp. 1121-1228.

[8] F. H. Clarke, Optimization and Nonsmooth Analysis, John Wiley \& Sons, New York, 1983.

[9] T. F. Cox and M. A. A. Cox, Multidimensional Scaling 2nd Ed, Chapman and Hall/CRC, 2001.

[10] J. Dattorro, Convex Optimization and Euclidean Distance Geometry. Meboo Publishing USA,2005. 
[11] C. Fortin and H. Wolkowicz, The trust region subproblem and semidefinite programming, Optim. Meth. Soft., (19) (2004), pp. 41-67.

[12] N. Gaffke and R. Mathar, A cyclic projection algorithm via duality, Metrika, 36 (1989), pp. 29-54.

[13] Y. Gao, Structured Low Rank Matrix Optimization Problems: a Penalty Approach, PhD Thesis (2010), National University of Singapore. Available from http://www.maths.edu.sg/ matsundf.

[14] W. Glunt, T. L. Hayden, S. Hong, and J. Wells, An alternating projection algorithm for computing the nearest Euclidean distance matrix, SIAM J. Matrix Anal. Appl., 11 (1990), pp. 589-600.

[15] W. Glunt, T. L. Hayden, and R. Raydan, Molecular conformations from distance matrices, J. Comput. Chem., 14 (1993), pp. 114-120.

[16] J. C. Gower, Properties of Euclidean and non-Euclidean distance matrices, Linear Algebra Appl., 67 (1985), pp. 81-97.

[17] T. L. Hayden and J. Wells, Approximation by matrices positive semidefinite on a subspace, Linear Algebra Appl., 109 (1988), pp. 115-130.

[18] N. Krislock and H. Wolkowicz, Euclidean distance matrices and applications, In Handbook of Semidefinite, Cone and Polynomial Optimization, M. Anjos and J. Lasserre (Editors), 2010.

[19] A. S. Lewis and M. L. Overton, Nonsmooth optimization via quasi-Newton methods, to appear in: Math. Program.

[20] L. Liberti, C. Lavor, N. Maculan, and A. Mucherino, Euclidean distance geometry and applications, available from arXiv:1205:0349v1. May 3, 2012.

[21] J. J. Moré, Generalizations of the trust region problems, Optim. Methods Soft., 2 (1993), pp. 189-209.

[22] M. L. Overton, Matlab package HANsO: Hybrid Algorithm for Non-Smooth Optimization. Available from: http://www.cs.nyu.edu/overton/software/hanso/

[23] H.-D. Qi, A semismooth Newton method for the nearest Euclidean distance matrix problem. SIAM J. Matrix Anal. Appl., 34 (2013), pp. 67-93.

[24] H.-D. Qi and X. M. Yuan, Computing the nearest Euclidean distance matrix with low embedding dimensions, Tech. Report, School of Mathematics, University of Southampton, September 2012.

[25] P. Stoica and J. Li, Source localization from range-difference measurements, IEEE Signal Processing Mag., 23 (2006), pp. 63-69.

[26] A. H. Sayed, A. Tarighat, and N. Khajehnouri, Network-based wireless location, IEEE Signal Processing Mag., 22 (2005), pp. 24-40.

[27] I. J. Schoenberg, Remarks to Maurice Fréchet’s article "Sur la définition axiomatque d'une classe d'espaces vectoriels distanciés applicbles vectoriellement sur l'espace de Hilbet”, Ann. Math. 36 (1935), pp. 724-732.

[28] K. C. Toh, An inexact path-following algorithm for convex quadratic SDP, Math. Program., 112 (2008), pp. 221-254.

[29] P. Tseng, Second-order cone programming relaxation of sensor network localization, SIAM J. Optim., 18 (2007), pp. $156-185$.

[30] G. Young and A. S. Householder, Discussion of a set of points in terms of their mutual distances, Psychometrika 3 (1938), pp. 19-22.

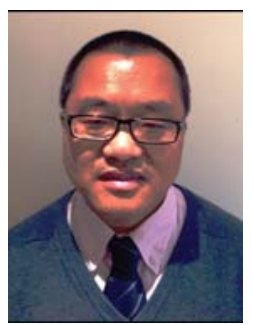

Houduo Qi received the BSc in Statistics from Peking University in 1990, MSc in Operational Research and Optimal Control from Qufu Normal University in 1993, and PhD in Operational Research and Optimal Control from Institute of Applied Mathematics, Chinese Academy of Sciences (CAS) in 1996.

From 1996 to 1998, he was a Chinese Postdoctoral Fellow at the Institute of Computational Mathematics, CAS. From 1998 to 2003, he was a research associate, research fellow and then the Australian Postdoctoral Fellow at The Hong Kong Polytecnique University and The University of New South Wales. In 2004, he was awarded Queen Elizabeth (QE) II Fellowship by the Australian Research Council. From September 2004, he joined the University of Southampton as a lecturer and then senior lecturer (since 2010) in operational research. He is mainly interested in Mathematical Optimization: classical nonlinear optimization, complementarity problems and variational inequalities; system of nonsmooth equations; stability analysis of neural networks; and matrix optimization with applications to finance and statistics. He has been an associate editor of Asia-Pacific Journal of Operational Research since 2011. From 2010, he has been a college member of Engineering and Physical Sciences Research Council, UK. 
This is the author's version of an article that has been published in this journal. Changes were made to this version by the publisher prior to publication.

The final version of record is available at http://dx.doi.org/10.1109/TSP.2013.2264814

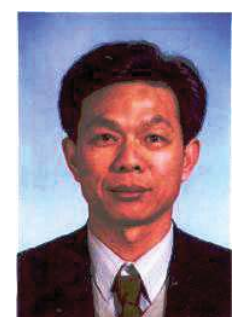

Naihua Xiu received the BSc in Mathematics from Hebei Normal University in 1982, and PhD in Operational Research and Optimal Control from Institute of Applied Mathematics, Chinese Academy of Sciences in 1997. From 1997 to 1999, he was a Chinese Postdoctoral Fellow at the Beijing Jiaotong University. From July 1999, he joined the Beijing Jiaotong University as an associate professor and then professor (since 2001) in operational research. He was also a Research Fellow of City University of Hong Kong from 2000 to 2002, and a Visiting Scholar of University of Waterloo from 2006 to 2007.

His research interest includes mathematical optimization, mathematics of operations research, and complementarity problems and variational inequalities. He is now the vice president of the Operations Research Society of China and the president of the Mathematical Programming Society of China, and serves as a member of editorial board for several journals such as Acta Mathematicae Applicatae Sinica, OR Transactions, and Operations Research and Management. He became a member of the National Basic Research Program of China (2010CB732501) from 2010.

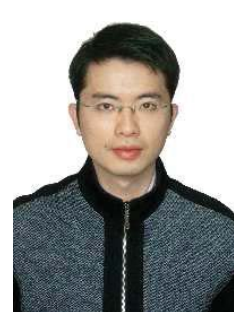

Xiaoming Yuan received the B.Sc. and M. Phil. degrees from Nanjing University in 1999 and 2001, respectively; and the Ph.D. degree from City University of Hong Kong in 2004. He was a post-doctoral fellow awarded by Pacific Institute of Mathematical Sciences in Canada in 2006. He is currently an assistant professor with Department of Mathematics, Hong Kong Baptist University. His research focuses on numerical optimization including variational inequalities and complementarity problems, convex programming, sparse and low-rank optimization. He is also interested in optimizations applications in areas such as image processing, statistical learning and operation management. 